\title{
Individual differences in electrodermal response conditioning and self-report of discomfort: A phobia analogue
}

\author{
SHEILA R. DEITZ \\ Colorado State University, Fort Collins, Colorado
}

\begin{abstract}
The present study was designed as an analogue to the acquisition and extinction of a classically conditioned phobic response. Thirty electrodermally labile and 30 electrodermally stabile nonphobic male undergraduates were assigned randomly to one of two experimental conditions, utilizing either "prepared" or "unprepared" CSs, after Seligman (1971). It was hypothesized that electrodermally labile subjects, in contrast to stabiles, would require a greater number of trials to habituate to either CS, would exhibit greater changes in log skin conductance during acquisition, would report higher verbal discomfort ratings related to the CS, and would require a greater number of trials to extinction. Differences between labiles and stabiles were also predicted for instrumental "escape" behavior during extinction. The results revealed significant differences between labile and stabile subjects on all predicted dimensions; however, no significant differences were found for the "prepared" versus "unprepared" CS contingencies. The study also reports unusual evidence for a relationship between a self-report measure of arousal and concomitant psychophysiological responsivity.
\end{abstract}

There has been considerable evidence to indicate that electrodermal responses (EDRs) provide valid indexes of a subject's autonomic response to noxious stimulation (see Katkin \& Deitz, 1973). Barlow, Agras, Leitenberg, and Wincze (1969) and Barlow, Leitenberg, Agras, and Wincze (1970) found differential EDRs by phobic and nonphobic subjects to the presentation of actual phobic stimuli. Geer (1966) demonstrated that spider-phobic subjects exhibited greater skin conductance responses (SCRs) to pictures of spiders than to stimuli neutral with respect to the spider phobia (pictures of snakes). Spider-phobic subjects also exhibited greater SCRs to pictures of spiders than did a matched control group that indicated no fear of spiders.

Lader (1967) and Lader, Gelder, and Marks (1967) found that specific phobics, in contrast to other neu-

This study is based on a doctoral dissertation submitted to the Department of Psychology, State University of New York at Buffalo, in partial fulfillment of the requirements for the $\mathrm{PhD}$ in clinical psychology. The author wishes to express her deep gratitude to her dissertation advisor, Edward S. Katkin, who continues to serve as an important mentor, gifted teacher, valued colleague, and friend. Appreciation is also expressed to the members of the author's doctoral committee, B. R. Bugelski, Walter Cohen, LeRoy H. Ford, Jr., John Lick, and Robert C. Nichols, for their many contributions to this research. Special thanks are also extended to Ronald McNish, Leslie Kahn, and Tedd Habberfield for their assistance in data collection. Requests for reprints should be sent to Sheila R. Deitz, Department of Psychology, Colorado State University, Fort Collins, Colorado 80523.

-Article accepted by previous editor, Richard F. Thompson rotic patients, resembled normal subjects in rate of electrodermal response habituation to auditory stimuli and in the number of spontaneous EDRs emitted. Furthermore, Lader (1967) noted a correlation between levels of general and overt anxiety and rate of EDR habituation to auditory stimuli, and he suggested that the presence of anxiety was associated with slower habituation. Lader and Wing (1966) reported similar findings: EDRs to auditory stimuli of patients with anxiety states showed less habituation than those of normals. The authors also found that highly anxious patients exhibited elevated levels of spontaneous EDR fluctuations.

Since Lacey and Lacey (1958) and Mundy-Castle and McKiever (1953) first pointed out that individual differences in the number of spontaneous EDRs reflected a reliable subject characteristic, much interest has been generated in utilizing measures of electrodermal lability (i.e., frequency of spontaneous electrodermal fluctuations) as a measure of individual differences in autonomic arousal. Electrodermal lability has been utilized as an individual difference measure of autonomic arousal (Silverman, Cohen, \& Shmavonian, 1959) and transitory response to stress (Katkin, 1965). Katkin and McCubbin (1969), operationally defining electrodermal lability as a measure of individual differences in anxiety, studied the habituation of EDRs to a moderately intense auditory stimulus. Electrodermally labile subjects did not habituate to the tone, whereas electrodermally stabile subjects did. Katkin and McCubbin (1969) thus provided support for the assumption that higher 
levels of autonomic arousal were associated with greater reactivity and delayed habituation to a moderately intense stimulus. Similarly, Koepke and Pribram (1966) reported slower habituation to tones presented at random intervals for labiles than for stabiles.

The relationship between individual differences in electrodermal activity and the acquisition of a conditioned response (CR) of fear remains to be investigated adequately. Hugdahl, Fredrikson, and Öhman (1977) found that both arousability, as defined by spontaneous EDRs, and stimulus content affected acquisition and resistance to extinction of a conditioned EDR. With this exception, however, few studies have investigated individual differences in the acquisition of a "fear" response in human subjects.

Recently, the analogy between the acquisition of conditioned fear in the laboratory and the acquisition of true phobias has been seriously questioned. Seligman $(1970,1971)$ raised important objections to the classical conditioning model of phobia acquisition and maintenance. He postulated a model of "preparedness" to account for the observation that phobias may be acquired in one trial and that certain stimuli are more likely than others to elicit a phobic response. By definition, prepared classical conditioning occurs "in one or a very few trials" and involves contingencies that "may have been intimately involved in the survival of the species"' (Seligman, 1971, p. 314).

The present experiment was designed to investigate the role of individual differences in autonomic reactivity in the acquisition and maintenance of a conditioned autonomic "fear" response, while considering also Seligman's (1971) "preparedness" notion. Utilizing electrodermal lability (frequency of spontaneous EDRs emitted during a rest period) as an individual difference measure of autonomic reactivity, the following hypotheses were tested. First, labiles were expected to require a greater number of trials than stabiles both to habituate to a neutral stimulus and to extinguish a CR to either of two conditioned stimuli (CSs). Two CSs were chosen to test Seligman's (1971) model of "preparedness." One CS, a live snake (.9 $\mathrm{m}$ in length) served as the "prepared" CS; the other stimulus, a rope equal in length and circumference to the snake, served as the "unprepared" CS.

Second, labiles were expected to show greater acquisition than stabiles when either CS was paired with shock. Verbal reports of discomfort associated with viewing either CS were expected to be higher for labile subjects than for stabiles. Significant differences between labile and stabile subjects were predicted both for the number of trials on which subjects would "escape" from either CS and the percentage of successful "escape" responses.

Finally, all subjects presented with the "prepared" CS were expected to require a greater number of trials to habituate, exhibit greater acquisition, report higher verbal discomfort ratings, and require more trials to extinguish a conditioned EDR than were subjects presented with the "unprepared" CS.

\section{METHOD}

\section{Subjects}

One hundred and twenty-two male undergraduates at the State University of New York at Buffalo served as subjects in the experiment. Of this number, 24 subjects were eliminated for failure to meet predetermined criteria on an abbreviated form of Wolpe and Lang's (1964) Fear Survey Schedule (FSS), 13 subjects refused to participate after having been informed that electric shock would be employed, and 5 subjects were eliminated due to equipment failure. The remaining 80 subjects were assigned randomly to one of two experimental conditions ("prepared" or "unprepared" CS) and to one of four experimenters. All subjects received credit toward a course requirement for their participation.

\section{Experimenters}

To control for sex bias, four experimenters (two females and two males) were used in the study. Of each sex, one experimenter was a graduate student engaged in psychophysiological research and one was an undergraduate familiar with both experimental design and laboratory procedures. All experimenters were trained individually prior to running subjects.

\section{Apparatus}

The experiment was conducted in a dimly illuminated, soundproof chamber, equipped with silent air conditioning and an intercom which allowed for communication between the subject and the experimenter. A speaker, placed next to the subject and connected to a tape recorder located outside the chamber, provided recorded instructions to the subject throughout the experiment. The subject was seated in a comfortable lounge chair, equipped with a red push button for use during the "escape" phase of the experiment. The push button was attached to the arm of the lounge chair and located on the subject's dominant side.

Skin conductance was detected by Beckman $\mathrm{Ag}-\mathrm{AgCl}$ electrodes (.64- $\mathrm{cm}^{2}$ surface area), with Beckman $\mathrm{NaCl}$ electrode paste as a contact medium. EDR electrodes were attached to the palm of the subject's nondominant hand.

A Grass Model 7 polygraph was located outside the chamber. Skin conductance was measured with a constant voltage skin conductance circuit designed by Lykken and Venables (1971) and fed into a Grass Model 7P1 preamplifier.

Inside the chamber, presentation of the CS was accomplished by placing the appropriate CS into a large gray wooden box $(56 \times$ $32 \times 61 \mathrm{~cm})$ with a one-way mirror $(51 \times 30 \mathrm{~cm})$ attached to the lower front portion of the box. Two 75-W floodlights were mounted inside the lid of the box and provided consistent illumination without distracting shadows. During CS presentations, the apparatus provided the subject with a clear view of the CS, whereas during interstimulus intervals, the subject could see only the mirror. The box was placed on a table, $.7 \mathrm{~m}$ in height, at a distance of $1.5 \mathrm{~m}$ from the subject.

The unconditioned stimulus (UCS) for all subjects was an electric shock of 1-sec duration, delivered through a silver-plated ring electrode placed on the subject's nondominant forefinger. The electrical source was a 6-V battery, fed through an astable multivibrator into an amplifier and transformer, producing a manipulable shock ranging from $0-70 \mathrm{~V}$. The shock apparatus was located outside the experimental chamber. The timing and sequence of the CS and UCS and of interstimulus intervals were controlled by two Hunter decade interval timers and by a Gerbrands filmstrip timer. To control for temporal conditioning, CSs were presented on a variable-interval schedule. Intertrial intervals ranged 
from $9 \mathrm{sec}$ to $19 \mathrm{sec}$, with a mean intertrial interval of $13.5 \mathrm{sec}$. The CS-UCS interval was set at $5 \mathrm{sec}$.

A series of relays, located outside the chamber, controlled the operation of the red push button mounted on the subject's chair during the escape phase of the experiment. If the subject pushed the button during the interval between CS onset and offset, the floodlights inside the box were extinguished, and the subject could no longer see the CS in the box. On the other hand, if the subject pushed the button at any time other than during this interval, the relay system rendered the push button inoperable on the next trial.

\section{Procedure}

Initial period. Upon arrival, the subject was presented with a set of written instructions and the FSS. The experimenter asked the subject to read the instructions and immediately left the room to permit the subject to decide if he wanted to participate in the study. The instructions stated that electric shock would be administered and that full participation credit would be granted to the subject, regardless of his decision to participate or leave.

Two items on the FSS (those tapping fear of harmless snakes and of enclosed places) were considered crucial for the present study. In order to insure that no actual phobics were included in the sample, any subject who indicated "a fair amount of anxiety" or greater on these items was dismissed from the experiment at this point. Any subject indicating "a little anxiety" or "no anxiety" on these items was invited into the laboratory. The subject was then escorted into the experimental chamber and seated in the lounge chair. At this point, alcohol was applied to the subject's palm and recording and shock electrodes were secured. Next, the subject was told: "Since each person reacts differently to electric shock, we are going to set a level of shock appropriate for you before we begin the experiment. We will begin with a very low level of shock, and I want you to tell me when you first feel the shock. Some people report a sensation of warmth or a tingle. Just let me know when you can detect the shock and describe what the sensation feels like to you."

For each subject, a level of shock that was rated by the subject as "uncomfortable, but not painful" was set, and the subject was informed that this level would remain constant throughout the experiment.

Adaptation period. The adaptation period lasted $10 \mathrm{~min}$, during which resting measures of tonic skin conductance and frequency of spontaneous EDRs were obtained. At the beginning of this time period, the subject received the following recorded instructions: "During the next ten minutes or so, we will just be recording your physiological responses. There will be no electric shock during this time period. Just sit back and relax." The total number of spontaneous EDRs emitted during the final 2 min of the adaptation period was determined for each subject and constituted one independent variable.

Habituation period. During the habituation period, the appropriate CS (snake or rope) was repeatedly presented to the subject in the absence of the UCS. The following recorded instructions were presented for this phase of the study: "During the next few minutes, the gray box in front of you will light up at various intervals and there will be no electric shock during this time period. Just watch the box." The habituation period continued until the subject reached a predetermined criterion of three successive presentations of the CS without exhibiting an SCR. For purposes of this experiment, an SCR was defined as a change in skin conductance of $.04 \mu \mathrm{mho}$ or greater.

Acquisition period. At the beginning of the acquisition phase, the subject received the following recorded instructions: "During the next time period, the box will continue to light up as before, but now you will also receive an electric shock at various times during this time period. Continue to watch the box." Following these instructions, the subject was presented with 3 trials of the CS without the UCS, to allow for some adaptation to the instructions. Next, the subject was presented with 10 delayed conditioning trials. The appropriate CS was presented $5 \mathrm{sec}$ prior to the onset of the moderately uncomfortable $1-\mathrm{sec}$ UCS, after which cessation of both CS and UCS occurred simultaneously. At this point, self-report ratings of discomfort related to viewing the CS were obtained. Using the intercom, the experimenter asked the subject: "(Subject's first name)?" (Wait for response.) "On a scale from 1 to 100 where 1 indicates 'no discomfort' and 100 indicates 'a great deal of discomfort,' please rate how you feel seeing the object in the box."

CS escape-extinction period. In order to provide an adequate analogue to a real phobic situation, the extinction phase of the experiment included an opportunity for the subject to "escape from" (i.e., not be forced to view) the CS that had been associated with a conditioned autonomic "fear" response. Thus, the final set of recorded instructions was presented to the subject: "During the next time period, the box will continue to light up as before, but now you can avoid seeing the object in the box by learning to make an appropriate response with the red button attached to the arm of your chair. From this point in the experiment on, there will be no electric shock. Continue watching the box." The "appropriate response" referred to in the final instructions required that the subject push the red button during the 6-sec interval between CS onset and CS cessation, and only during this interval. A correct response instantaneously shut off the floodlights in the box, so that the subject could see only the mirror.

The criterion for extinction was identical to that used during habituation. Thus, the subject was presented with the CS in the absence of the UCS until he exhibited "no response" on three successive trials, regardless of whether or not his escape behavior had extinguished. At this point, the experimenter entered the chamber, debriefed the subject, and dismissed him from the experiment.

Identifying labiles and stabiles. A median split procedure was used to differentiate electrodermally labile from electrodermally stabile subjects. Of the original 80 subjects, the 20 subjects scoring in the middle range of frequency of spontaneous EDRs emitted during the final 2 min of the adaptation period (overall median $=18$ ) were eliminated from the sample, leaving a total of 60 subjects equally distributed across each of the experimental groups. The mean number of spontaneous EDRs for labiles was 25.3 (range: 19 to 38 ); for stabiles, the mean was 7.3 (range: 1 to 15).

\section{RESULTS}

\section{Habituation to Initial Presentations of the CS}

The mean number of trials to habituation, with standard deviations, for each of the four experimental groups is presented in Table 1 . An analysis of variance performed on these data revealed a significant main effect for lability $[F(1,56)=23.30, p<$ $.001]$. Labiles required a greater number of trials to habituate to the CS than did stabiles. No significant effects were found for either the snake-rope dimension or for the interaction between lability and CS.

\section{Acquisition Period}

All subjects received 10 trials of CS-UCS pairing during acquisition. Skin conductance was measured

Table 1

Mean Number of Trials to Habituation, With Standard Deviations, for the Four Experimental Groups

\begin{tabular}{lcr}
\hline \multicolumn{1}{c}{ Group } & Mean & SD \\
\hline Labile-Snake & 31.93 & 14.89 \\
Labile-Rope & 31.20 & 26.34 \\
Stabile-Snake & 12.20 & 10.32 \\
Stabile-Rope & 10.80 & 7.82 \\
\hline
\end{tabular}


Table 2

Mean Amplitude of Change in Log Skin Conductance $(\times 1,000)$ for the Four Experimental Groups During the Acquisition Period

Trial Number

\begin{tabular}{lccccccccccc} 
& \multicolumn{1}{c}{ Group } & 1 & 2 & 3 & 4 & 5 & 6 & 7 & 8 & 9 \\
\hline Labile-Snake & 20.50 & 41.11 & 39.55 & 32.62 & 42.03 & 34.69 & 33.70 & 36.66 & 26.86 & 35.60 \\
Labile-Rope & 23.09 & 31.08 & 38.90 & 36.59 & 27.86 & 29.80 & 41.42 & 36.69 & 38.75 & 38.87 \\
Stabile-Snake & 18.16 & 30.04 & 30.57 & 33.95 & 18.59 & 28.18 & 14.12 & 14.86 & 21.99 & 14.02 \\
Stabile-Rope & 15.98 & 33.52 & 27.35 & 20.89 & 17.74 & 12.80 & 17.24 & 14.13 & 14.05 & 8.70 \\
\hline
\end{tabular}

directly, and conditioned responses to the CS were defined as increases in skin conductance of $.04 \mu \mathrm{mho}$ or greater, with a latency of 1 to $3 \mathrm{sec}$ after CS onset. The mean amplitude of change in log skin conductance $(\times 1,000)$ for the four experimental groups for Trials $1-10$ is presented in Table 2 . Analysis of these data revealed a significant main effect for lability $[F(1,56)=10.11, \mathrm{p}<.005]$ and a significant main effect for trials $[F(9,504)=2.87, p<.05]$, as well as a significant interaction between lability and trials $[F(9,504)=2.05, p<.05]$. The interaction is depicted in Figure 1.

\section{Verbal Ratings of Discomfort}

Immediately following the acquisition period and prior to extinction, all subjects were asked to rate,

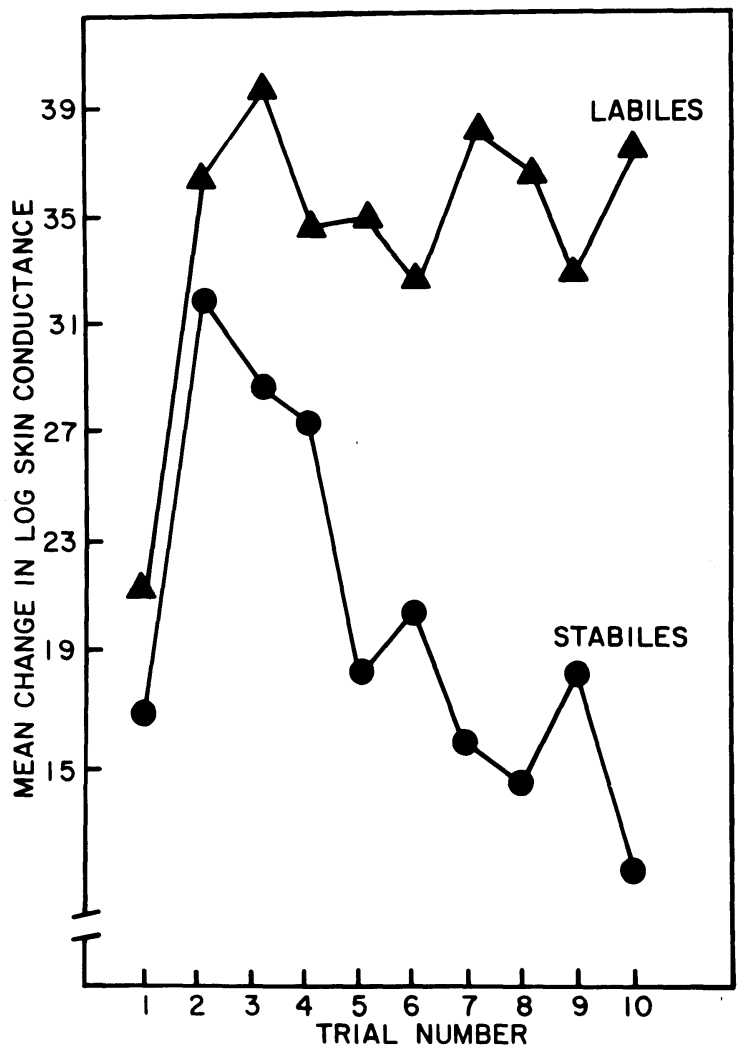

Figure 1. Mean amplitude of change in log skin conductance $(\times 1,000)$ for labiles and stabiles during the acquisition period.
Table 3

Mean Verbal Ratings of Discomfort at Seeing the CS, With Standard Deviations, for the Four Experimental Groups

\begin{tabular}{lrr}
\hline \multicolumn{1}{c}{ Group } & Mean & \multicolumn{1}{c}{ SD } \\
\hline Labile-Snake & 15.07 & 15.23 \\
Labile-Rope & 26.07 & 34.13 \\
Stabile-Snake & 10.47 & 10.86 \\
Stabile-Rope & 5.40 & 9.80 \\
\hline
\end{tabular}

on a scale from 1 to 100 , their discomfort at seeing the CS. The mean verbal discomfort ratings, with standard deviations, for the four experimental groups, are presented in Table 3. Analysis of these data again revealed a significant main effect for lability $[F(1,56)$ $=5.94, \mathrm{p}<.025$ ], but nonsignificant results for the snake-rope dimension, for the lability $\times$ CS interaction, and for differences between the two labile groups. Thus, in addition to exhibiting larger SCRs during acquisition, labiles reported greater discomfort related to viewing the CS than did stabile subjects.

\section{Extinction Period}

The number of trials required to achieve a criterion of three successive trials of "no response," as measured by changes in skin conductance, was obtained for each subject. The mean number of trials to extinction, with standard deviations, for each of the four experimental groups is presented in Table 4. Paralleling the habituation data, analyses revealed a significant main effect for lability $[F(1,56)=8.98$, $\mathrm{p}<.005$ ], but nonsignificant results for all other analyses. Thus, labiles required a greater number of trials to extinguish, once the CR was acquired, than did stabiles.

\section{Analysis of Escape Data}

The number of escape trials, defined as the number of trials during extinction on which the subject successfully "escaped" from the CS, is presented in Table 5. Analyses of these data revealed no significant differences between the groups on any of the dimensions investigated. The percentages of successful escape responses (in comparison with the total number of buttonpresses) for all of the four groups are also reported in Table 5. An analysis of variance performed on these data revealed a significant main 
Table 4

Mean Number of Trials to Extinction, With Standard Deviations, for the Four Experimental Groups

\begin{tabular}{lll}
\hline \multicolumn{1}{c}{ Group } & Mean & SD \\
\hline Labile-Snake & 43.73 & 34.81 \\
Labile-Rope & 33.07 & 26.61 \\
Stabile-Snake & 18.00 & 16.89 \\
Stabile-Rope & 19.00 & 17.27 \\
\hline
\end{tabular}

Table 5

Mean Number of Escape Trials and Percentages of Escape Responses, With Standard Deviations, for the Four Experimental Groups

\begin{tabular}{lcc}
\hline \multicolumn{1}{c}{ Group } & Mean & SD \\
\hline & Number of Escape Trials \\
Labile-Snake & 12.47 & 37.74 \\
Labile-Rope & 10.67 & 17.38 \\
Stabile-Snake & 9.73 & 18.10 \\
Stabile-Rope & 12.13 & 16.26 \\
& Percentage of Escape Responses \\
& 14.20 & 26.27 \\
Labile-Snake & 27.19 & 29.61 \\
Labile-Rope & 43.31 & 41.87 \\
Stabile-Snake & 40.51 & 41.51 \\
Stabile-Rope & &
\end{tabular}

effect for lability $[F(1,56)=5.35, p<.025]$, indicating that stabile subjects "escaped" from the CS on a greater percentage of extinction trials than did labiles.

\section{DISCUSSION}

\section{Individual Differences in Electrodermal Lability During Habituation and Extinction}

The finding that labile subjects required a greater number of trials to habituate to the CS than did stabiles is consistent with the literature and lends support to the findings of Katkin (1975) and Lader (1967). Similarly, the finding that labiles required a greater number of trials to extinction of the $C R$ than stabiles was as predicted and appears to reflect a phenomenon similar to that of labiles' greater resistance to habituation.

\section{Acquisition}

The hypothesis that labiles would show greater changes in amplitude of $\log \mathrm{SC}$ than stabiles during the acquisition stage was confirmed. Although consistent with a learning theory prediction that arousal would facilitate learning of a classically conditioned response, this finding poses several theoretical problems. Lader (1967) and Lader, Gelder, and Marks (1967) demonstrated that specific phobics, in contrast to other neurotic patients, resembled normal subjects on measures of electrodermal activity. However, the present results, indicating that the more highly aroused subjects (labiles) exhibited greater acquisition of conditioned autonomic responses than did subjects lower in arousal (stabiles), appear to conflict with these earlier findings. It may be that extremely reactive individuals acquire a learned response (phobia) as an adaptive coping mechanism and thus exhibit EDR patterns similar to nonphobic individuals.

The finding, for the acquisition period, of a significant main effect for trials and a significant trials $\times$ lability interaction revealed that both labiles and stabiles increased in response strength after the first pairing of the CS and UCS, that is, between Trial 1 and Trial 2, and that labiles maintained a stable level of conditioning. In contrast, stabiles appear to have habituated to both the CS and UCS after the second conditioning trial.

\section{Verbal Report Ratings of Discomfort}

The hypothesis that labiles would report higher discomfort ratings than stabiles when viewing the CS after acquisition "was confirmed. Frequently, lack of agreement between various response indexes of anxiety has been reported, particularly when selfreport and psychophysiological measures of arousal have been compared (Katkin, 1975; Krause, 1961; Martin, 1961). Thus, the present study presents unusual evidence for a relationship between a self-report measure of discomfort and concomitant psychophysiological responsivity. Mandler, Mandler, and Uviller (1958) reported that subjects scoring higher on the Manifest Anxiety Scale (Taylor, 1953) indicated higher estimates of their autonomic activity than did low-scoring subjects. However, their verbal report measures were obtained postexperimentally, not during the actual experiment.

The finding that verbal ratings of discomfort were related to the presence of the CS alone is also of theoretical importance. In a study designed to investigate the dynamics of a phobic reaction, it is essential to show that the CS alone, after pairing with the UCS, acquires negative valence for the subject. Once the fear response has been established in a "true" phobic pattern, the UCS is of little importance. The presence of the CS alone is sufficient to cause a phobic to experience fear and discomfort that motivates avoidance behavior, resulting in a relatively permanent absence of the UCS.

\section{Individual Differences in Escape Behavior}

The hypothesis that labile and stabile subjects would respond differently during the escape phase of the experiment was only partially confirmed. The finding that stabiles exhibited a greater percentage of successful escape trials than did labiles is unusual. Individuals exhibiting a "true" phobic reaction would be expected to escape from the fear-eliciting CS more rapidly and with greater efficiency than nonphobic individuals. In the present experiment, the analogy is clear: Since labiles exhibited both acquisition and 
maintenance of a conditioned autonomic fear response and reported greater discomfort at viewing the CS than did stabiles, labiles would be expected to escape from the CS more readily than stabiles. It is possible that the instructions to the subjects in the present study neutralized the expected results. Koenig and Del Castillo (1969) and Wickens, Allen, and Hill (1963) studied the influence of instructional set on extinction behavior and reported that instructions to subjects which indicated "no more shock" generally accelerated the extinction process without producing immediate extinction.

\section{Lack of Evidence Supporting "Preparedness"' Theory}

In contrast to previous studies (e.g., Fredrikson \& Öhman, 1979; Öhman, Eriksson, \& Oloffson, 1975; Öhman, Erixon, \& Löfberg, 1975), none of the hypotheses designed to test Seligman's theory of "preparedness" was confirmed in the present study. According to Seligman (1971), "Prepared-learning provides a better fit with phobias than unprepared learning because we have reason to believe that it (1) can be acquired in one trial, (2) is selective, (3) is resistant to extinction, and (4) may be noncognitive"' (p. 314). In examining the present acquisition data, it must be noted that a significant increase in response amplitude was noted for both labiles and stabiles after one trial. Thus, without investigating the differential effects of conditioning over several trials, one might argue that one-trial learning did, indeed, occur. Seligman offers no explanation for the observation that two different individuals, both experiencing a traumatic event associated with a "selective" stimulus, may develop quite different responses to the stimulus. Whereas one individual may become phobic, the other may not. Although immediate and consistent contact with the fearful object in the absence of pain or anxiety could certainly be an important factor in determining these different reactions, individual differences in physiological arousal might well be another.

In the present study, hypotheses designed to test Seligman's (1971) emphasis on the selective nature of the CS postulated that subjects presented with the "prepared" CS (snake) would require a greater number of trials to habituate, would exhibit greater acquisition, and would require a greater number of trials to extinguish a CR than would subjects presented with the "unprepared" CS (rope). However, the present data revealed no differential responding to "prepared" and "unprepared" stimulus dimensions and, thus, provided no support for Seligman's model of phobia acquisition.

\section{REFERENCES}

Barlow, D. H., \& Agras, W. S., Leitenberg, H., \& Wincze, J. P. An experimental analysis of the effectiveness of "shaping" in reducing maladaptive avoidance behaviour: An analogue study. Behaviour Research and Therapy, 1970, 8, 165-173.

Barlow, D. H., Leitenberg, H., Agras, W. S., \& Wincze, J. $P$. The transfer gap in systematic desensitization: An analogue study. Behaviour Research and Therapy, 1969, 7, 191-196.

Fredrikson, M., \& Öhman, A. Cardiovascular and electrodermal responses conditioned to fear-relevant stimuli. Psychophysiology, 1979, 16, 1-7.

GEe R, J. H. Fear and autonomic arousal. Journal of Abnormal Psychology, 1966, 71, 253-255.

Hugdahl, K., Fredrikson, M., \& Öhman, A. "Preparedness" and "arousability" as determinants of electrodermal conditioning. Behaviour Research and Therapy, 1977, 15, 345-353.

KATKIN, E. S. Relationship between manifest anxiety and two indices of autonomic response to stress. Journal of Personality and Social Psychology, 1965, 2, 324-333.

KATKIN, E. S. Electrodermal lability: A psychophysiological analysis of individual differences in response to stress. In I. G. Sarason \& C. D. Spielberger (Eds.), Stress and anxiety. Washington, D.C: Hemisphere Publishing, 1975.

KatKIn, E. S., \& DEITz, S. R. Systematic desensitization. In W. F. Prokasy \& D. C. Raskin (Eds.), Electrodermal activity in psychological research. New York: Academic Press, 1973.

KATKIn, E. S., \& McCubbin, R. J. Habituation of the orienting response as a function of individual differences in anxiety and autonomic lability. Journal of Abnormal Psychology, 1969, 74, 54-60.

Koenig, K. P., \& Del Castillo, D. False feedback and longevity of the conditioned GSR during extinction: Some implications for aversion therapy. Journal of Abnormal Psychology, 1969, 74, 505-510.

Koepke, J. E., \& Pribram, K. H. Habituation of the GSR as a function of stimulus duration and spontaneous activity. Journal of Comparative and Physiological Psychology, 1966, 61, 442-448.

KraUse, M. S. The measurement of transitory anxiety. Psychological Review, 1961, 68, 178-189.

LACEY, J. I., \& LACEY, B. C. The relationship of resting autonomic activity to motor impulsivity. Research Publications of the Association for Nervous and Mental Diseases, 1958, 36, 144-209.

LADER, M. H. Palmar skin conductance measures in anxiety and phobic states. Journal of Psychosomatic Research, 1967, 11, 271-281.

Lader, M. H., Gelder, M. G., \& Marks, I. M. Palmar skin conductance measures as predictors of response to desensitization. Journal of Psychosomatic Research, 1967, 11, 283-291.

Lader, M. H., \& Wing, L. Physiological measures, sedative drugs, and morbid anxiety. London: Oxford University Press, 1966.

LyKKen, D. T., \& Venables, P. H. Direct measurement of skin conductance: A proposal for standardization. Psychophysiology, 1971, 52, 629-634.

Mandler, G., Mandler, J. M., \& Uviller, E. T. Autonomic feedback: The perception of autonomic activity. Journal of $A b$ normal and Social Psychology, 1958, 56, 367-373.

Martin, B. The assessment of anxiety by physiological behavioral measures. Psychological Bulletin, 1961, 58, 234-255.

Mundy-Castle, A. C., \& McKiever, B. L. The psychophysiological significance of the galvanic skin response. Journal of Experimental Psychology, 1953, 46, 15-23.

Öhman, A., Eriksson, A., \& Olofsson, C. One-trial learning and superior resistance to extinction of autonomic responses conditioned to potentially phobic stimuli. Journal of Comparative and Physiological Psychology, 1975, 88, 619-627.

Öhman, A., Erixon, G., \& Löfberg, I. Phobias and preparedness: Phobic versus neutral pictures as conditioned stimuli for human autonomic responses. Journal of Abnormal Psychology, $1975,84,41-45$.

Seligman, M. E. P. On the generality of the laws of learning. Psychological Review, 1970, 77, 406-418. 
Seligman, M. E. P. Phobias and preparedness. Behavior Therapy, 1971, 2, 307-320.

Silverman, A. J., Cohen, S. I., \& Shmavonian, B. M. Investigation of psychophysiologic relationships with skin resistance measures. Journal of Psychosomatic Research, 1959, 4, 65-87.

TAYLOR, J. A. A personality scale of manifest anxiety. Journal of Abnormal and Social Psychology, 1953, 48, 285-290.

Wickens, D. D., Allen, C. K., \& Hill, F. A. Effect of instruc- tions and UCS strength on extinction of the conditioned GSR Journal of Experimental Psychology, 1963, 66, 235-240.

WolPE, J., \& LANG, P. H. A fear survey schedule for use in behaviour therapy. Behaviour Research and Therapy, 1964, 2, 27-30.

(Manuscript received January 5, 1981; revision accepted for publication August 11, 1981.) 